Pulsar Astronomy -...2000 and Beyond

ASP Conference Series, Vol. 202,2000

$M$. Kramer, $N$. Wex, and $R$. Wielebinski, eds.

\title{
Deconfinement signals from pulsar timing
}

\author{
Hovik Grigorian, Gevorg Poghosyan and Edvard Chubarian \\ Physics Department, Yerevan State University, Alex Manoogian Str. 1, \\ 375025 Yerevan, Armenia \\ David Blaschke \\ Fachbereich Physik, Universität Rostock, Universitätsplatz 1, D-18051 \\ Rostock, Germany
}

\begin{abstract}
The occurence of a quark matter core in rotating compact stars has been investigated within general relativity as a function of both the rotational frequency and the total baryon number. We demonstrate that the deviation of the braking index from $n=3$ signals not only the occurence but also the size of a quark matter core in a pulsar. We suggest that in systems with mass accretion onto a rapidly rotating compact star a spin-down to spin-up transition might signal a deconfinement transition in its interior.
\end{abstract}

Recently it has been suggested that the deconfinement transition in rapidly rotating compact stars could have observable consequences in pulsar timing since phase transitions in the dense stellar interior could result in changes of the rotational behavior (Glendenning, Pei, \& Weber 1997; Grigorian, Hermann, \& Weber 1999). Further constraints for the nuclear equation of state come from the observation of quasi-periodic brightness oscillations (QPO's) in low-mass $\mathrm{X}$-ray binaries which entail mass and radius limits for rapidly rotating neutron stars (Lamb, Miller, \& Psaltis 1998).

We apply the method of perturbation theory (Hartle 1967; Hartle \& Thorne 1968; Sedrakian \& Chubarian 1968) which is the most transparent and systematic approach to the problem of stationary gravitational fields and their sources and has been proven successful in general relativity as well as in alternative theories of gravitation (Grigorian \& Chubarian 1985).

We have calculated the mass, angular momentum and shape deformation from the iterative solution of the gravitational field equations in case of hydrodynamical, thermodynamical and chemical equilibrium for given total baryon number and angular velocity $\Omega$ of the object with a model equation of state describing the deconfinement transition (Chubarian et al. 1999).

The moment of inertia changes predominantly due to matter redistribution and shape deformation rather than gravitational fields and rotational energy and reflects the occurrence of a quark matter core (Chubarian et al. 1999). Observable consequences of the onset of deconfinement are changes in the braking index for isolated pulsars, see Fig. 1 (a), and a flip from spin-down to spin-up behavior, see Fig. I (b), for systems with mass accretion at constant angular momentum $J$. 

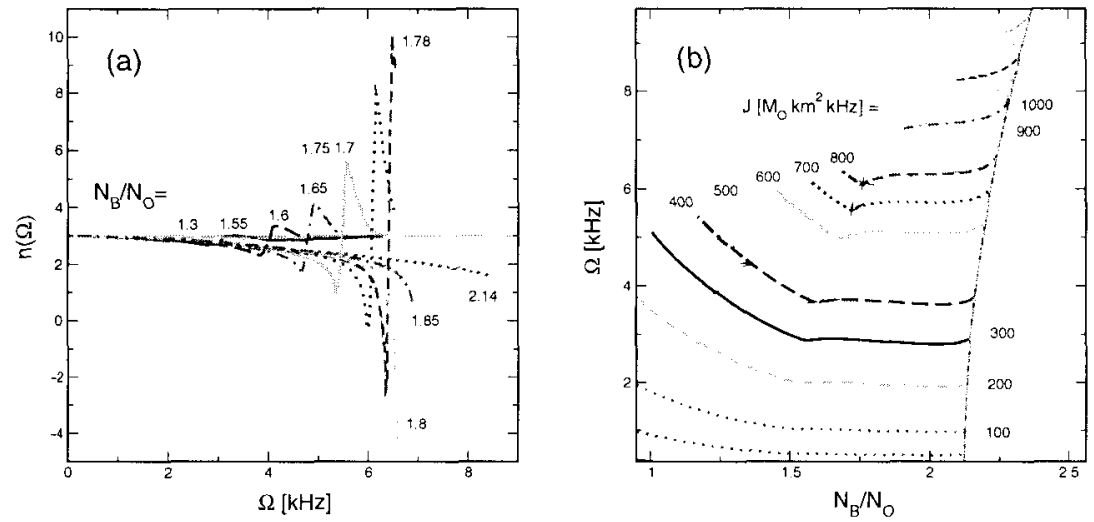

Figure 1. (a) Braking index $n(\Omega)$ for configurations with different total baryon numbers. Deviation from $n=3$ indicates the occurrence of a quark matter core correlated with its size. (b) Spin-down to spinup transition for mass accretion at constant angular momentum $J$ as a function of the baryon number $N_{B}$ accumulated in the compact star.

Comparison of our results (Fig. 1 (a)) with those of Glendenning, Pei \& Weber (1997) shows that deconfinement signals from pulsar timing are very sensitive to the choice of the EOS and we have therefore started QCD based studies of the EOS at finite baryon density and temperature for applications to compact stars (Blaschke et al. 1999).

This work has been supported by the Volkswagen Stiftung under grant no. $\mathrm{I} / 71226$ and by a stipend from the DAAD.

\section{References}

Glendenning, N. K., Pei, S., \& Weber, F. 1997, Phys. Rev. Lett., 79, 1603

Grigorian, H., Hermann, M., \& Weber, F. 1999, Phys. At. Nucl., 30, 380

Lamb, F. K., Miller, M. C., \& Psaltis, D. 1998, Rapid X-Ray Variability of Neutron Stars in Low-Mass Binary Systems, astro-ph/9802089

Hartle, J. B. 1967, Ap. J., 150, 1005;

Hartle, J. B., \& Thorne, K. S. 1968, Ap. J. , 153, 807

Sedrakian, D. M., \& Chubarian, E. V. 1968, Astrofizika, 4, 239, 551

Grigorian, H., \& Chubarian, E. V. 1985, Astrofizika, 23, 177

Chubarian, E., Grigorian, H., Poghosyan, G., \& Blaschke, D. 1999, astro$\mathrm{ph} / 9903489$

Blaschke, D., Grigorian, H., Poghosyan, G., Roberts, C. D., \& Schmidt, S. 1999, Phys. Lett. B, 450, 207 